\title{
The role of carboplatin in the neoadjuvant chemotherapy treatment of triple negative breast cancer
}

\author{
Aurelio Bartolome Castrellon, ${ }^{1}$ Ihor Pidhorecky, ${ }^{2}$ Vicente Valero, ${ }^{3}$ Luis Estuardo Raez ${ }^{4}$ \\ ${ }^{1}$ Breast Cancer Research, Memorial Cancer Institute, Memorial Healthcare System, Hollywood, FL; ${ }^{2}$ Surgical \\ Oncology Services \& Pancreaticobiliary Center, Memorial Cancer Institute, Memorial Healthcare System, Hollywood, \\ FL; ${ }^{3}$ Department of Breast Medical Oncology, Division of Cancer Medicine, The University of Texas MD Anderson \\ Cancer Center, Houston, TX ; ${ }^{4}$ Hematology/Oncology and Medical Director of Memorial Cancer Institute, Memorial \\ Healthcare System, Hollywood, FL, USA
}

\begin{abstract}
Triple negative breast (TNBC) cancer constitutes a heterogeneous group of disease with histologic and molecular differences. Complete pathologic response to neoadjuvant chemotherapy (NACT) in TNBC is associated with improved outcomes. Efforts have been made in identifying drug combinations that will increase the response rate to preoperative chemotherapy. In this review we present recent studies that have incorporated carboplatin $(\mathrm{Cb})$ in the NACT of TNBC. We discuss the homologous recombination deficiency score and the somatic or germline mutation for BRCA as potential biomarkers for future selection of patients that could benefit from the addition of $\mathrm{Cb}$ to NACT.
\end{abstract}

\section{Introduction}

Triple-negative breast cancer (TNBC) accounts for approximately 20 percent of breast cancers (BC) diagnosed worldwide, representing almost 200,000 cases each year. ${ }^{1}$ Epidemiologic studies illustrate a high prevalence of TNBC among younger women, when compared to the other BC subtypes. These patients are also at higher risk to develop brain or visceral metastasis. ${ }^{2-5}$ In addition, it appears to be more common among black woman than whites, and is associated with the BRCA1 genetic mutation. 6,7 TNBC is characterized by the absence of expression of the estrogen (ER), progesterone receptors (PR) and lack of amplification of the human epidermal growth factor receptor 2 (HER2)/Neu gene. ${ }^{8}$

Correspondence: Aurelio Bartolome Castrellon, Breast Cancer Research, Memorial Cancer Institute, Memorial Healthcare System, Hollywood, FL, USA.

E-mail: acastrellon@mhs.net

Key words: Breast cancer; neoadjuvant chemotherapy; platinum agents.

Received for publication: 8 November 2016.

Revision received: 24 February 2017.

Accepted for publication: 27 February 2017.

This work is licensed under a Creative Commons Attribution NonCommercial 4.0 License (CC BY-NC 4.0).

(C) Copyright A.B. Castrellon et al. 2017

Licensee PAGEPress, Italy

Oncology Reviews 2017; 11:324

doi:10.4081/oncol.2017.324
Unlike hormonal receptor positive (HR+) and HER2 overexpressing breast cancers, TNBC is unresponsive to endocrine therapy and HER2-targeted agents and treatment options are limited to conventional cytotoxic chemotherapy. ${ }^{9}$ Chemotherapy has been effective in the treatment of early-stage disease, with pathologic complete response (pCR) rates exceeding those of $\mathrm{HR}+$ subtypes. ${ }^{10,11}$ Patients with metastatic disease however experience rapid progression through several lines of chemotherapy, and overall survival (OS) in the metastatic setting is usually poor with reports being between 9 and 13 months. ${ }^{12}$ Pathologic complete response rates to neoadjuvant chemotherapy (NACT) among patients with TNBC range from $27-45 \%$, while pCR rate for patients with HER2 negative/HR+ breast cancer is generally around $10-20 \% .{ }^{13,14}$ Pathological complete response has been proposed as a surrogate endpoint for prediction of long-term clinical benefit, such as disease free survival (DFS) and OS. ${ }^{14,15}$ However, while patients with TNBC who achieve a pCR appear to have a good DFS, patients with TNBC who have more than minimal residual disease at surgery have a much higher risk of early distant disease recurrence. ${ }^{16-18}$

Based on the fact that currently there are no approved targeted therapies for the neoadjuvant or palliative treatment of TNBC, identifying potential targets and developing effective targeted agents is greatly needed.

\section{Heterogeneity of TNBC}

It is well recognized that there are histologic and molecular differences in TNBC. ${ }^{19}$ From the histology point of view, the majority of TNBC corresponds to the invasive ductal carcinoma type (IDC). Other less commonly seen histologies include: Medullary carcinoma, metaplastic carcinoma, adenoid cystic carcinoma and apocrine carcinoma. ${ }^{20}$ The prognosis varies greatly among these different histology groups. Patients with metaplastic carcinoma have been identified to have higher relapse rates; a retrospective study by Bae and colleagues, demonstrated an inferior 3 year DFS in patients with lymph node metastasis who underwent adjuvant chemotherapy of $44.4 \%$ vs $72.5 \%$ when compared to TNBC-IDC $(\mathrm{P}=0.025) .{ }^{21}$ Medullary carcinomas on the other hand, are believed to have a better prognosis. This was demonstrated in an analysis of 13 International Breast Cancer Study Group (IBCSG) trials, where the 14 year DFS was $89 \%$ for patients with medullary carcinoma (ER negative and high grade tumors) vs $63 \%$ for patients with TNBC-IDC (HR 0.24, $\mathrm{P}=0.002){ }^{22}$ Adenoid cystic carcinomas have also been found to have a good prognosis with 5 year DFS typically above $90 \% .^{23}$

The triple negative clinical subtype comprises mainly the 
basal-like molecular subtype, but caution should be used when referring to TNBC in general as "basal like" tumors. As an example, 172 triple-negative tumors based on IHC staining were correlated with gene expression profiles that defined the basal subtype and only $71 \%$ of TNBC were consistent with the basal subtype. ${ }^{24}$ At the molecular level, gene expression (GE) profiles from 587 TNBC cases by cluster analysis identified 6 TNBC types displaying unique $\mathrm{GE}$ and ontologies, including 2 basal-like (BL 1 and $\mathrm{BL}$ 2 ), an immunomodulatory (IM), a mesenchimal (M), a mesenchimal stem-like (MSL), and a luminal androgen receptor (LAR) subtype. ${ }^{25}$ BL 1 and BL 2 subtypes have higher expression of cell cycle and DNA damage response genes, and representative cell lines that preferentially respond to platinum agents. The IM subtype is enriched for immune cell processes. M and MSL subtypes are enriched in GE for epithelial-mesenchymal transition and growth factor pathways, cell models of this the subtype responded to NVP-BEZ235 (a PI3K/mTOR inhibitor) and dasatinib (an $\mathrm{abl} / \mathrm{scr}$ inhibitor). The LAR subtype includes patients with decreased relapse-free survival and is characterized by androgen receptor (AR) signaling. LAR cell lines were uniquely sensitive to bicalutamide (an AR antagonist). ${ }^{25}$

\section{Recent NACT trials with $\mathrm{Cb}$ in TNBC}

There is a large body of literature indicating that patients with aggressive breast cancer subtypes who obtain a pCR to NACT have a better prognosis; this is especially true for the hormonal receptor negative (HR-) BC subtypes. ${ }^{17,18}$ Currently pCR is considered a surrogate endpoint for OS in patients receiving NACT for TNBC. The optimal chemotherapy regimen however remains to be determined. TNBC demonstrates sensitivity to DNA-damaging agents like platinum. ${ }^{10}$ Based on this finding a number of clinical trials have sought to determine if adding $\mathrm{Cb}$ to anthracycline-taxane based or simply taxane NACT would increase the pCR rates (Table 126-34).

In the phase II GeparSixto trial 315 patients with stage II to III TNBC were treated for 18 weeks with weekly paclitaxel (wP) 80 $\mathrm{mg} / \mathrm{m}^{2}$ and non-pegylated-liposomal doxorubicin $20 \mathrm{mg} / \mathrm{m}^{2}$. Bevacizumab $15 \mathrm{mg} / \mathrm{kg}$ every 2 weeks (q $2 \mathrm{w}$ ) was given concomitantly. All patients were randomized 1:1 to receive concurrently $\mathrm{Cb}$ AUC 2 but later on reduced to 1.5 secondary to toxicity. Primary outcome of the study was pCR rates. ${ }^{26}$ The addition of $\mathrm{Cb}$ increased pCR from $37 \%$ in the control group to $53 \%$ in patients that received $\mathrm{Cb}(\mathrm{P}=0.005)$. Hematological side effects were more common in the $\mathrm{Cb}$ group and included grade $\geq 3$ neutropenia $65 \%$ vs $27 \%$, grade $\geq 3$ anemia $15 \%$ vs $<1 \%$ and grade $\geq 3$ thrombocy- topenia $14 \%$ vs $1 \%$. Cb was more often associated with dose discontinuation, in $48 \%$ with $\mathrm{Cb}$ and $39 \%$ without $\mathrm{Cb}(\mathrm{P}=0.031) .{ }^{26}$ The 3 year analysis shows that $85.8 \%$ of the patients treated with $\mathrm{Cb}$ were without evidence of disease $v s 76.1 \%$ in the control group (HR 0.56, 95\% CI 0.33-0.96, $\mathrm{P}=0.0350$ ). ${ }^{27}$

In the randomized phase II trial conducted by the Cancer Leukemia Group (CALGB 40603), 443 patients with stage II to III TNBC received a backbone chemotherapy of wP $80 \mathrm{mg} / \mathrm{m}^{2}$ for 12 weeks, followed by doxorubicin plus cyclophosphamide $\mathrm{q} 2 \mathrm{w}$ (ddAC) for four cycles and were randomly assigned to concurrent Cb AUC 6 every 3 weeks ( 3 w) for four cycles and/or bevacizumab $10 \mathrm{mg} / \mathrm{kg} \mathrm{q} 2 \mathrm{w}$ for nine cycles. ${ }^{28}$ Employing one-sided $\mathrm{P}$ values, addition of either $\mathrm{Cb}(60 \%$ vs $44 \% ; \mathrm{P}=0.0018)$ or bevacizumab (59\% vs 48\%; $\mathrm{P}=0.0089)$ significantly increased pCR in the breast, whereas only $\mathrm{Cb}(54 \%$ vs $41 \%$; $\mathrm{P}=0.0029)$ significantly raised pCR in the breast and axilla. Patients assigned to either $\mathrm{Cb}$ or bevacizumab were less likely to complete wP and ddAC without skipped doses, dose modification, or early discontinuation resulting from toxicity. Grade $\geq 3$ neutropenia and thrombocytopenia were more common with $\mathrm{Cb}$, as were hypertension, infection, thromboembolic events, bleeding, and postoperative complications with bevacizumab. ${ }^{28}$ The analysis of event free survival (EFS) and OS with a median follow-up duration of 39 months, showed that treatment with $\mathrm{Cb}$ or bevacizumab did not significantly affect either outcome. The addition of $\mathrm{Cb}$ was associated with an EFS hazard ratio (HR) of $0.84(95 \% \mathrm{CI} 0.58-1.22, \mathrm{P}=0.36)$ and a survival HR of 1.15 (95\% CI $0.74-1.79, \mathrm{P}=0.53)$. Outcomes were similar with the addition of bevacizumab. ${ }^{29}$

The ISPY-2, randomized 60 women whose tumors had a genomic signature consistent with TNBC to receive wP $80 \mathrm{mg} / \mathrm{m}^{2}$ for 12 weeks, followed by ddAC for four cycles, with or without an experimental regimen consisting of $\mathrm{Cb}$ AUC $6 \mathrm{q} 3 \mathrm{w}$ for four cycles and the oral poly-ADP ribose polymerase (PARP) inhibitor, veliparib (50 mg twice daily by mouth). ${ }^{30}$ The study demonstrated a pCR of $51 \%$ in the veliparib-Cb containing arm [ $95 \%$ probability interval (PI) 36-66\%] compared to $26 \%$ in the control arm (95\% PI $9-43 \%)$. Given the design of the study, it is difficult to determine how much the addition of the PARP-inhibitor added to the effect of $\mathrm{Cb}$. Early detection of therapy response or resistance in the neoadjuvant setting may help to optimize the chemotherapy strategy. In the phase II Adjuvant Dynamic Marker-Adjusted Personalized Therapy (ADAPT) triple negative trial, 336 patients with centrally confirmed TNBC were randomized to receive nab-paclitaxel at $125 \mathrm{mg} / \mathrm{m}^{2}$ either with Cb AUC 2 or Gemcitabine $1000 \mathrm{mg} / \mathrm{m}^{2} .^{31}$ The study reported pCR of $45.9 \%$ vs $28.7 \%$, favoring the Cb containing arm. Early response was predictive of pCR regardless of the treatment arm. The observed efficacy in this study seems com-

Table 1. Selected Cb NACT trials in TNBC.

\begin{tabular}{|c|c|c|c|c|c|c|}
\hline $\begin{array}{l}\text { Study } \\
\text { [reference] }\end{array}$ & $\begin{array}{l}\text { Study } \\
\text { design }\end{array}$ & $\begin{array}{l}\text { Chemotherapy } \\
\text { regimen }\end{array}$ & $\mathbf{N}$ & $\begin{array}{c}\text { PCR } \\
\text { definition } \\
\text { used }\end{array}$ & $\begin{array}{c}\text { PCR } \\
(\%) \\
\text { Control }\end{array}$ & $\begin{array}{c}\text { PCR } \\
(\%) \\
\text { Platinum }\end{array}$ \\
\hline GeparSixto ${ }^{26}$ & Randomized phase II & $\mathrm{wP}+\mathrm{nPLD} 20 \mathrm{mg} / \mathrm{m}^{2} \mathrm{qw}+\mathrm{B} 15 \mathrm{mg} / \mathrm{kg} \mathrm{q} 3 \mathrm{w} \pm$ Cb AUC $1.5-2 \mathrm{qw}$ x $18 \mathrm{w}$ & 315 & урT0 урN0 & 37 & 53 \\
\hline CALGB $40603^{28}$ & Randomized phase II & wP x $12 \pm$ Cb AUC 6 q 3w x $4 \rightarrow$ ddAC x $4 \pm$ B 10 mg/kg q 2w x 9 & 433 & ypT0/is ypN0 & 41 & 54 \\
\hline ISPY-230 & Randomized phase II & $\mathrm{wP} \times 12 \pm \mathrm{Cb}$ AUC $6 \mathrm{q} 3 \mathrm{w}$ x $4+$ veliparib $\rightarrow \operatorname{ddAC}$ x 4 & 60 & ypT0/is ypN0 & 26 & 51 \\
\hline ADAPT $^{31}$ & Randomized phase II & $\begin{array}{l}\text { weekly nap-paclitaxel } 125 \mathrm{mg} / \mathrm{m}^{2}+\text { Cb AUC } 2 \\
\text { or gemcitabine } 1,000 \mathrm{mg} / \mathrm{m}^{2} \text { on day } 1 \text { and } 8 \mathrm{q} 3 \mathrm{w} \times 4\end{array}$ & 336 & ypT0/is ypN0 & 28.7 & 45.9 \\
\hline Sharma et al. ${ }^{34}$ & Observational & Cb AUC 6 + Docetaxel 75 mg/m² $3 \mathrm{w}$ x 4-6 cycles & 76 & ypT0/is ypN0 & na & 66 \\
\hline
\end{tabular}

Abbreviations: AC, doxorubicin $60 \mathrm{mg} / \mathrm{m}^{2}$ and cyclophosphamide $600 \mathrm{mg} / \mathrm{m}^{2}$; ddAC, dose dense AC; Cb, carboplatin; AUC, area under the curve; B, Bevacizumab; wP, weekly paclitaxel $80 \mathrm{mg} / \mathrm{m}^{2} ; \mathrm{nPLD}$, non-pegylated-liposomal doxorubicin; pCR, complete pathologic response; na, not available; qw, every week; q $2 \mathrm{w}$, every 2 weeks; q $3 \mathrm{w}$, every 3 weeks; ypT0 ypN0, absence of invasive cancer and in situ cancer in the breast and axillary nodes; ypT0/is ypN0, absence of invasive cancer in the breast and axillary nodes, irrespective of carcinoma in situ. 
parable to longer and less tolerable anthracycline-taxane containing regimens. Patients that did not achieve a pCR in the study were offered standard post-operative chemotherapy with epirubicin and cyclophosphamide for 4 cycles. It is unknown if outcome is affected by the type of chemotherapy administered in order to obtain a $\mathrm{pCR}$. Anthracyclines are associated with long-term worrisome side effects, especially cardiotoxicity and leukemia. ${ }^{32}$ There have been studies looking at omitting these agents in the adjuvant treatment of TNBC, but so far it has been demonstrated that 6 cycles of docetaxel in combination with cyclophosphamide is associated with a higher breast cancer recurrence, when compared to standard anthracycline-taxane based regimens. ${ }^{33}$ This raises the question if results could be improved by combining docetaxel with $\mathrm{Cb}$ instead. A prospective multisite registry study evaluated Docetaxel in combination with $\mathrm{Cb}$ and included 76 patients with $\geq \mathrm{T} 1 \mathrm{c}$ to Stage III TNBC. Patients received 4-6 cycles of docetaxel 75 $\mathrm{mg} / \mathrm{m} 2$ in combination with $\mathrm{Cb}$ AUC 6 given $\mathrm{q} 3 \mathrm{w}$. This regimen produced pCR in $66 \%$ of the patients. With a median follow up of 2.3 years the cohort of patients that achieve a pCR demonstrated a $95 \%$ recurrence free survival. ${ }^{34}$

\section{Can we select patients that benefit from the addi- tion of Cb to NACT?}

Gene defects in the homologous recombination (HR) pathway are of potential therapeutic relevance in a variety of cancers. Clinical studies have demonstrated that BRCA1/2-deficient tumors are sensitive to both platinum salts and PARP-inhibitors. ${ }^{35,36}$ The three DNA-based homologous recombination deficiency (HRD) scores: HRD-loss of heterozygosity score (LOH), HRD-telomeric allelic imbalance score (TAI), and HRD-large-scale state transition score (LST) are highly correlated with defects in BRCA1/2, and are associated with response to platinum therapy in triple negative breast and ovarian cancer. ${ }^{37-40}$

Analysis of triple negative tumors in the GeparSixto clinical trial found HR deficiency in $136(70.5 \%)$ tumors; $82(60 \%)$ of them showed high HRD score (LOH score + TAI score + LST score $\geq 42$ ) without BRCA mutation. The study utilized the HRD assay developed by Myriad Genetics Inc. (Salt lake City, UT, https://www.myriad.com). HR deficiency was associated with a higher rate of pCR $55.9 \%$ vs $29.8 \%(\mathrm{P}=0.001)$. Adding carboplatin $\mathrm{Cb}$ to the paclitaxel, non-pegylated-liposomal doxorubicin and bevacizumab combination increased the $\mathrm{pCR}$ rate from $45.2 \%$ to $64.9 \%$ in HR deficient tumors $(\mathrm{P}=0.025)$. This effect was also seen in patients with somatic BRCA mutations, where the $\mathrm{pCR}$ rate was increased from $38.1 \%$ to $69.7 \%$ with the addition of $\mathrm{Cb}(\mathrm{P}=0.022)$. The pCR rate in the HR non-deficient patients was $20 \%$ without $\mathrm{Cb}$ and $40.7 \%$ with $\mathrm{Cb}$, but did not reach statistical significance $(\mathrm{P}=0.146) .{ }^{41}$

Pooled analysis of six phase II clinical trials (including GeparSixto), in which patients with TNBC received a platinum agent, demonstrated that patients with high HRD score were significantly more likely to achieve a pCR than those with HR-nondeficient tumors: $53 \%$ vs $18 \%$ (adjusted odds ratio $=4.64$; $\mathrm{P}<0.0001$ ), regardless of BRCA1/2 mutation status. ${ }^{42}$ To further support the fact that the presence of a BRCA-1 mutation confers high sensitivity to platinum agents, Byrski and colleagues treated 107 patients with BRCA-1 associated breast cancer with single agent cisplatin $75 \mathrm{mg} / \mathrm{m}^{2}$ every 3 weeks for 4 cycles. The study demonstrated a very significant pCR rate of $61 \%$, considering anthracyclines and taxanes were not given. ${ }^{43}$

Masuda and colleagues evaluated clinical outcomes in 130 patients based on subtypes of TNBC. ${ }^{16}$ They found that patients with the basal-like 1 subtype had the highest pCR rate $(52 \%)$. In contrast, those with the LAR subtype had one of the lowest pCR rates $(10 \%)$. However, despite their low pCR rate, OS was better in patients with the LAR subtype. ${ }^{16}$ These findings indicate that perhaps the LAR molecular subtype of TNBC may not benefit from more intense NACT protocols that add $\mathrm{Cb}$.

Although specific tests are not approved or commercially available at the moment, it is possible that in the future, the NACT agents could be tailored according to the molecular subtype of TNBC. Adding Cb could be more beneficial in subtypes other than the LAR. It is also possible that addition of $\mathrm{Cb}$ could at some point be selected based on high HRD scores or the presence of a somatic or germline mutation for BRCA.

\section{Prognostic significance of $\mathrm{pCR}$ in TNBC}

Evidence from accumulated neoadjuvant studies reveals that pCR provides a surrogate marker that is predictive of long-term clinical response and survival in TNBC patients. ${ }^{14,15}$ Despite its widespread use, there is still no uniform definition of pCR. Three definitions have been traditionally used by different investigators: i) ypT0 ypN0: absence of invasive cancer and in situ cancer in the breast and axillary nodes; ii) ypT0/is ypN0: absence of invasive cancer in the breast and axillary nodes, irrespective of carcinoma in situ; iii) ypT0/is: absence of invasive cancer in the breast, irrespective of ductal carcinoma in situ or nodal involvement.

Two large meta-analyses have looked at the long-term outcomes of patients achieving pCR after NACT. Both studies have demonstrated a major benefit in the long-term outcome from achieving a pCR in patients with aggressive BC subtypes (triplenegative; HR-/HER2-positive and high-grade HR+/HER2-negative). ${ }^{17,18}$

In the German Breast Group (GBG) and the Arbeitsgemeinschaft Gynäkologische Onkologie Breast (AGO-B) study groups, seven prospective clinical trials with a total of 6377 patients receiving neoadjuvant anthracycline-taxane-based chemotherapy were analyzed during a median follow up of 46.3 months. Prognostic impact of pCR on DFS was demonstrated in 4193 patients according to the breast cancer intrinsic subtype. The eradication of tumor from both breast and lymph nodes (ypT0/is ypN0 and ypT0 ypN0) compared to the absence of tumor in breast only (ypT0/is) revealed a stronger association with improved EFS and OS. TNBC represented $15 \%$ of the study group and demonstrated a pCR (ypT0 ypN0) of 44\%. Progression free survival in this subgroup of patients with pCR was over $90 \%$ at 5 years $(\mathrm{P}<0.001) .{ }^{17}$

The US Food and Drug Administration established an international working group known as Collaborative Trials in Neoadjuvant Breast Cancer (CTNeoBC). The study included 12 international neoadjuvant trials with 11,955 patients in the pooled responder analysis. Patients who achieved a pCR had longer EFS and OS than did patients with residual invasive cancer. Eradication of tumor from both the breast and axillary lymph nodes (ypT0pN0 and ypT0/is ypN0) was better associated with improved EFS and OS than was eradication of invasive tumor from the breast alone (ypT0/is). The association between pCR and long-term outcomes was strongest in patients with TNBC (EFS: HR 0.24, 95\% CI 0.18-0.33; OS: $0.16,0.11-0.25) .{ }^{18}$ However, the trial-level association between $\mathrm{pCR}$ and long-term outcome by tumor subtype recorded no correlation between improvement in frequency of pCR and the treatment's effect on EFS or OS. It is possible that dif- 
ferent biological subtypes of $\mathrm{BC}$ require a different end point definition regarding $\mathrm{pCR}$ to indicate a survival benefit and the inclusion of heterogeneous populations may have obscured the association. It has also been indicated that large increases in $\mathrm{pCR}$ between the control group and investigation arm will be needed in NACT studies to demonstrate a statistically significant change in survival. ${ }^{44,45}$ This maybe the reason behind the fact that improvement in pCR by $20 \%$ in the case of the Neo-ALLTO trial, narrowly missed statistical significance in the ALLTO trial (HR 0.84, $97.5 \%$ CI $0.70-1.02){ }^{46,47}$

\section{Selected ongoing Cb NACT studies in TNBC}

There are several studies evaluating various schedules and combinations of $\mathrm{Cb}$ in the NACT of TNBC (Table 2). The phase II NeoStop clinical trial determines the need for anthracyclines in the NACT setting by randomizing patients to a non-anthracycline containing arm of Docetaxel and $\mathrm{Cb}$ in standard dose and frequency given for six cycles vs. weekly paclitaxel in combination with $\mathrm{Cb}$ followed by ddAC. The study's primary endpoint is pCR rates (NCT02413320). The phase III PEARLY clinical trial is randomizing patients to receive a taxane-anthracycline chemotherapy plus or minus $\mathrm{Cb}$, in either the neoadjuvant or adjuvant setting. The primary outcome of the study is five-year EFS, secondary outcomes include pCR rates and long-term effects of Cb (NCT02441933).

The 50-gene qPCR assay (PAM50) can identify the intrinsic biological BC subtypes using RNA isolated from more readily available formalin-fixed, paraffin-embedded (FFPE) tissue. These subtypes can also be assessed using a multiplexed gene-expression profiling technology (NanoString Technologies; Seattle, WA, USA). The PAM50 gene set provides a risk of relapse score not only in ER-positive, node negative patients (similarly to the Oncotype Dx Recurrence Score) but also in the ER negative disease. Additionally, the PAM50 assay is highly predictive of neoadjuvant response when considering all BC subtypes. ${ }^{48}$ This test is being used to identify predictors of response to NACT with docetaxel and $\mathrm{Cb}$ (NCT01560663). The GeparOla multicenter, prospective, randomized, open-label phase II clinical trial, is testing the effect of adding olaparib to weekly paclitaxel and $\mathrm{Cb}$ followed by epirubin and cyclophosphamide (NCT02789332). Patients will have centrally confirmed tumor high HRD score and known germline BRCA and/or tumor BRCA mutation. The study is looking at pCR rates and assessing the effect of olaparib in this population of patients. Immune checkpoint inhibitors have demonstrated activity as single agents in the treatment of advanced
TNBC. ${ }^{49}$ The effect is potentiated by the addition of nab-paclitaxel. ${ }^{50}$ To explore this effect in NACT of TNBC, the randomized clinical trial NeoTRIPaPDL1aims to evaluate the addition of atezolizumab to $\mathrm{Cb}$ and nab-paclitaxel in patients with locally advanced TNBC (NCT02620280).

\section{Conclusions}

The long-term survival effect of the addition of $\mathrm{Cb}$ to standard NACT regimens remains unclear. The 3 year follow up of the GeparSixto clinical trial demonstrated an EFS advantage favoring the $\mathrm{Cb}$ containing arm, while the CALGB40603 39 month median follow up report did not show a statistical difference in EFS or OS with the addition of $\mathrm{Cb}$. It is important to understand that neither one of these two studies were powered to demonstrate EFS differences. Since there are no targeted therapies currently approved for the NACT of TNBC, we need to continue to rely on chemotherapies with the goal of increasing pCR rates. Based on the fact that pCR confers a good prognosis, it seems reasonable to continue to seek this outcome. The improvement in pCR seen in these trials however, comes at the cost of increased toxicity, dose reductions and omissions, which were needed in up to $40-50 \%$ of the patients. Ongoing randomized phase III clinical trials will hopefully provide more information on the survival effect, as well as on long-term toxicity with the addition of $\mathrm{Cb}$ to adjuvant chemotherapy (NCT02488967, NCT02441933).

Based on the fact that TNBC constitutes a heterogeneous group of disease, it is important to point out that future studies will need to individualize therapies according to the different subgroups of TNBC. Current studies have started to evaluate the addition of $\mathrm{Cb}$ to NACT based on high HRD scores. Other studies test its addition to patients with molecular profiling consistent with the basal subtype. Once the patients that are likely to benefit from the addition of $\mathrm{Cb}$ to NACT are identified, this may result in improved response to treatment demonstrated by higher rates of $\mathrm{pCR}$. Most importantly, patients that are not likely to benefit will be spared from the additional toxicity of $\mathrm{Cb}$.

Until more information is available, the addition of $\mathrm{Cb}$ to standard NACT for TNBC should be individualized. Currently it is acceptable to add it in the following cases: BRCA-associated $\mathrm{BC}$, patients with inflammatory $\mathrm{BC}$ or for those who present with locally advanced disease. Patients should be healthy enough and clinically fit to tolerate the increased toxic effect of adding $\mathrm{Cb}$ to standard NACT. At this time, treating TNBC patients with NACT, which does not incorporates anthracyclines, remains investigation-

Table 2. Selected active NACT evaluating the addition of carboplatin in TNBC.

\begin{tabular}{|c|c|c|c|}
\hline $\begin{array}{l}\text { NCT Identifier } \\
\text { (Acronym) }\end{array}$ & Phase & Study design & Chemotherapy regimen \\
\hline $\begin{array}{l}\text { NCT02413320 } \\
\text { NeoSTOP }\end{array}$ & II & Randomized, open-label & wP x $12+$ Cb AUC 6 q 3w x $4 \rightarrow$ ddAC x 4 vs Docetaxel 75 mg $/ \mathrm{m}^{2}+$ Cb AUC 6 q 3w x 6 \\
\hline $\begin{array}{l}\text { NCT02441933 } \\
\text { PEARLY }\end{array}$ & III & Randomized, open-label & $\mathrm{AC} \times 4 \mathrm{q} 3 \mathrm{w} \rightarrow$ taxane (Docetaxel $75 \mathrm{mg} / \mathrm{m}^{2} \mathrm{q} 3 \mathrm{w} \times 4$ or wP x 12) \pm Cb AUC 5 q 3w x 4 \\
\hline $\begin{array}{l}\text { NCT02789332 } \\
\text { GeparOla }\end{array}$ & II & Randomized, open-label & wP + olaparib 100 mg bid x 12 w or Cb AUC 2 q w x $12 \rightarrow$ EC q 2-3w x 4 \\
\hline NCT01560663 & II & Observational, case control & Docetaxel $75 \mathrm{mg} / \mathrm{m}^{2}+$ Cb AUC $6 \mathrm{q} 3 \mathrm{w}$ x 6 \\
\hline $\begin{array}{l}\text { NCT02620280 } \\
\text { NeoTRIPaPDL1 }\end{array}$ & II & Randomized, open- label & Cb AUC 2 + nab-paclitaxel $125 \mathrm{mg} / \mathrm{m}^{2}$ on day 1 and $8 \mathrm{q} 3 \mathrm{w}$ x $8 \pm$ atezolizumab $1200 \mathrm{mg}$ i.v. on day $1 \mathrm{q} 3 \mathrm{w}$ x 8 \\
\hline
\end{tabular}


al. If possible, patients should be enrolled in ongoing $\mathrm{Cb}$ NACT studies looking to answer the questions raised above.

\section{References}

1. Trivers KF, Lund MJ, Porter PL, et al.The epidemiology of triple-negative breast cancer, including race. Cancer Causes Control 2009;20:1071.

2. Foulkes WD, Smith IE, Reis-Filho JS. Triple-negative breast cancer. N Engl J Med 2010;363:1938-48.

3. Lin NU, Claus E, Sohl J, Razzak AR, et al. Sites of distant recurrence and clinical outcomes in patients with metastatic triple-negative breast cancer. Cancer 2008;113:2638-45.

4. Hernandez-Aya LF, Chavez-Macgregor M, Lei X, et al. Nodal status and clinical outcomes in a large cohort of patients with triple-negative breast cancer. J Clin Oncol 2011;29:2628-34.

5. Dent R, Trudeau M, Pritchard KI, et al. Triple-negative breast cancer: clinical features and patterns of recurrence. Clin Cancer Res 2007;13:4429-34.

6. Gonzalez-Angulo AM, Timms KM, Liu S, et al. Incidence and outcome of BRCA mutations in unselected patients with triple receptor-negative breast cancer. Clin Cancer Res 2011;17: 1082-9.

7. Hartman AR, Kaldate RR, Sailer LM, et al. Prevalence of BRCA mutations in an unselected population of triple-negative breast cancer. Cancer 2012;118:2787-95.

8. Hammond ME, Hayes DF, Dowsett M, et al. American Society of Clinical Oncology/College Of American Pathologists guideline recommendations for immunohistochemical testing of estrogen and progesterone receptors in breast cancer. J Clin Oncol 2010;28:2784.

9. Isakoff SJ. Triple-negative breast cancer: role of specific chemotherapy. Cancer J 2010;16:53-61.

10. von Minckwitz G, Martin M. Neoadjuvant treatments for triple-negative breast cancer (TNBC). Ann Oncol 2012;23: vi35-9.

11. Amos KD, Adamo B, Anders CK. Triple-negative breast cancer: An update on neoadjuvant clinical trials. Int J Breast Cancer 2012;2012:385978.

12. Kassam F, Enright K, Dent R, et al. Survival outcomes for patients with metastatic triple-negative breast cancer: implications for clinical practice and trial design. Clin Breast Cancer 2009;9:29-33.

13. Carey LA, Dees EC, Sawyer L, et al. The triple negative paradox: primary tumor chemosensitivity of breast cancer subtypes. Clin Cancer Res 2007;13:2329-34.

14. Liedtke C, Mazouni C, Hess KR, et al. Response to neoadjuvant therapy and long-term survival in patients with triple-negative breast cancer. J Clin Oncol 2008;26:1275-81.

15. Mieog JS, van der Hage JA, van de Velde CJ. Preoperative chemotherapy for women with operable breast cancer. Cochrane Database Syst Rev 2007;2:CD005002.

16. Dent R, Hanna WM, Trudeau M, et al. Time to disease recurrence in basal-type breast cancers: effects of tumour size and lymph node status. Cancer 2009;115:4917-23.

17. von Minckwitz G, Untch M, Blohmer JU, et al. Definition and impact of pathologic complete response on prognosis after neoadjuvant chemotherapy in various intrinsic breast cancer subtypes. J Clin Oncol 2012;30:1796-804.

18. Cortazar P, Zhang L, Untch M, et al. The CTNeoBC pooled analysis. Lancet 2014;384:164-72.

19. Constantinidou A, Jones RL, Reis-Filho JS. Beyond triple-negative breast cancer: the need to define new subtypes. Expert
Rev Anticancer Ther 2010;10:1197-213.

20. Ishikawa Y, Horiguchi J, Toya H, et al. Triple-negative breast cancer: Histological subtypes and immunohistochemical and clinicopathological features. Cancer Scic 2011;102:656-62.

21. Bae SY, Lee SK, Koo MY, et al. The prognoses of metaplastic breast cancer patients compared to those of triple-negative breast cancer patients. Breast Cancer Res Treat 2011;126:4718.

22. Huober J, Gelber S, Goldhirsch A, et al. Prognosis of medullary breast cancer: analysis of 13 International Breast Cancer Study Group (IBCSG) trials. Ann Oncol 2012;23:2843-51.

23. Kulkarni N, Pezzi CM, Greif JM, et al. Rare breast cancer: 933 adenoid cystic carcinomas from the National Cancer Data Base. Ann Surg Oncol 2013;20:2236-41.

24. Bertucci F, Finetti P, Cervera N, et al. How basal are triple-negative breast cancers? Int J Cancer 2008;123:236.

25. Lehmann BD, Bauer JA, Chen X, et al. Identification of human triple-negative breast cancer subtypes and preclinical models for selection of targeted therapies. J Clin Invest 2011;121:2750.

26. von Minckwitz G, Schneeweiss A, Loibl S, et al. Neoadjuvant arboplatin in patients with triple-negative and HER2-positive early breast cancer (GeparSixto; GBG 66): a randomised phase 2 trial. Lancet Oncol 2014;15:747.

27. von Minckwitz G, Loibl S, Schneeweiss A, et al. Early survival analysis of the randomized phase II trial investigating the addition of carboplatin to neoadjuvant therapy for triple-negative and HER2-positive early breast cancer (GeparSixto). SABCS 2015; abstr S2-04.

28. Sikov WM, Berry DA, Perou CM, et al. Impact of the addition of carboplatin and/or bevacizumab to neoadjuvant once-perweek followed by dose-dense doxorubicin and on pathologic complete response rates in stage II to III triple-negative breast cancer: CALGB 40603 (Alliance). J Clin 2015;33:13.

29. Sikov WM, Berry DA, Perou CM, et al. Event-free and overall survival following neoadjuvant weekly paclitaxel and dosedense $\mathrm{AC}+/$ - carboplatin and/or bevacizumab in triple-negative breast cancer: Outcomes from CALGB 40603 (Alliance). SABC 2015; abstr S2-05.

30. Rugo HS, Olopade OI, DeMichele A, et al. Adaptive Randomization of Veliparib-Carboplatin Treatment in Breast Cancer. N Engl J Med 2016;375:23-34.

31. Gluz O, Nitz U, Liedtke C, et al. Comparison of 12 weeks neoadjuvant nab-paclitaxel combined with carboplatinum vs. gemcitabine in triple-negative breast cancer: WSG-ADAPT TN randomized phase II trial. SABCS 2015; abstr S6-07

32. Valentini CG, Fianchi L, Voso MT, et al. Incidence of acute myeloid leukemia after breast cancer. Mediterr J Hematol Infect Dis 2011;3:e2011069.

33. Blum JL, Flynn PJ, Yothers G, et al. Interim joint analysis of the $\mathrm{ABC}$ (anthracyclines in early breast cancer) phase III trials (USOR 06-090, NSABP B-46I/USOR 07132, NSABP B-49 [NRG Oncology]) comparing docetaxel + cyclophosphamide (TC) $\mathrm{v}$ anthracycline/taxane-based chemotherapy regimens (TaxAC) in women with high-risk, HER2-negative breast cancer. ASCO 2016; Abstr 1000.

34. Sharma P, Kimler BF, Ward C, et al. Prognoses of triple negative breast cancer patients who attain pathological complete response with neoadjuvant carboplatin/docetaxel and do not receive adjuvant anthracycline chemotherapy. J Clin Oncol 2016;34: abstr 1015.

35. Konecny GE, Kristeleit RS. PARP inhibitors for BRCA1/2mutated and sporadic ovarian cancer: current practice and 
future directions. Br J Cancer 2016;115:1157-73.

36. Telli ML, Timms K, Reid JE, et al. Combined Homologous Recombination Deficiency (HRD) scores and response to neoadjuvant platinum-based chemotherapy in triple-negative and/or BRCA1/2 mutation-associated breast cancer. ASCO 2015.

37. Abkevich V, Timms KM, Hennessy BT, et al. Patterns of genomic loss of heterozygosity predict homologous recombination repair defects in epithelial ovarian cancer. Br J Cancer 2012;107:1776-82.

38. Birkbak NJ, Wang ZC, Kim J-Y, Eklund AC, et al. Telomeric allelic imbalance indicates defective DNA repair and sensitivity to DNA damaging agents. Cancer Discov 2012;2:366-75.

39. Popova T, Manie E, Rieunier G, et al. Ploidy and large-scale genomic instability consistently identify basallike breast carcinomas with BRCA1/2 inactivation. Cancer Res 2012;72:545462.

40. Timms KM, Abkevich V, Hughes E, et al. Association of BRCA1/2 defects with genomic scores predictive of DNA damage repair deficiency among breast cancer subtypes. Breast Cancer Res 2014;16:475.

41. von Minckwitz G, Timms K, Untch M, et al. Prediction of pathological complete response (pCR) by Homologous Recombination Deficiency (HRD) after carboplatin-containing neoadjuvant chemotherapy in patients with TNBC: Results from GeparSixto. J Clin Oncol 2015;33: abstr 1004.

42. Telli ML, Timms KM, Reid J, et al. Homologous recombination deficiency (HRD) as a predictive biomarker of response to neoadjuvant platinum-based therapy in patients with triple negative breast cancer. SABCS 2015; abstr P3-07-12.

43. Byrski T, Huzarski T, Dent R, et al. Pathologic complete response to cisplatin in BRCA1-positive breast cancer patients. Breast Cancer Res Treat 2014;147:401.

44. Masuda H, Baggerly KA, Wang Y, et al. Differential response to neoadjuvant chemotherapy among 7 triple-negative breast cancer molecular subtypes. Clin Cancer Res 2013;19:5533-40.

45. Pennisi A, Kieber-Emmons T, Makhoul I, et al. Relevance of Pathological Complete Response after Neoadjuvant Therapy for Breast Cancer. Breast Cancer (Auckl) 2016;10:103-6.

46. Baselga J, Bradbury I, Eidtmann H, et al. Lapatinib with trastuzumab for HER2-positive early breast cancer (NeoALTTO): a randomised, open-label, multicentre, phase 3 trial. Lancet 2012;379:633-40.

47. Piccart-Gebhart MJ, Holmes AP, Baselga J, et al. First results from the phase III ALTTO trial (BIG 2-06; NCCTG [Alliance] N063D) comparing one year of anti-HER2 therapy with Lapatinib alone (L), trastuzumab alone (T), their sequence ( $\mathrm{T}$ then $\mathrm{L}$ ), or their combinations $(\mathrm{T}+\mathrm{L})$ in the adjuvant treatment of HER2-positive early breast cancer (EBC) [abstract] J Clin Oncol 2014;32:LBA4.

48. Liu MC, Pitcher BN, Mardis ER, et al. PAM50 gene signatures and breast cancer prognosis with adjuvant anthracycline- and taxane-based chemotherapy: correlative analysis of C9741 (Alliance) npj. Breast Cancer 2016;2:15023.

49. Nanda R, Chow LQ, Dees EC, et al. Pembrolizumab in patients with advanced triple-negative breast cancer: Phase Ib KEYNOTE-012 study. J Clin Oncol 2016 [Epub ahead of print].

50. Adams S, Diamond J, Hamilton E, et al. Safety and clinical activity of atezolizumab (anti-PDL1) in combination with nabpaclitaxel in patients with metastatic triple-negative breast cancer. J Clin Oncol 2016;34: abstr 1009. 